J. Astrophys. Astr. (2000) 21, 373-377

\title{
The Current Status of Kinematic Solar Dynamo Models
}

\author{
Arnab Rai Choudhuri, Department of Physics, Indian Institute of Science, Bangalore \\ 560012, India \\ e-mail:arnab@physics.iisc.ernet.in
}

\begin{abstract}
This review provides a historical overview of how research in kinematic solar dynamo modeling evolved during the last few decades and assesses the present state of research. The early pioneering papers assumed the dynamo to operate in the convection zone. It was suggested in the 1980s that the dynamo operates in a thin layer at the bottom of the convection zone. Some researchers in recent years are arguing that the poloidal field is produced near the surface-an idea that goes back to Babcock (1961) and Leighton (1969).
\end{abstract}

Key words. Sun-MHD-dynamo theory.

\section{Introduction}

The aim of solar dynamo theory is to explain how solar magnetic fields are generated and maintained by the nonlinear interactions between the solar plasma and magnetic fields. In the full dynamo problem, therefore, one has to study how the magnetic field $\mathbf{B}(\mathbf{x}, t)$ and the velocity field $\mathbf{v}(\mathbf{x}, t)$ act on each other. This is a problem which can be attacked only numerically in any non-trivial situation and is a formidable problem even by the standard of today's computers. This led to the development of kinematic dynamo theory, in which one assumes the velocity field to be 'given' and studies only the behaviour of the magnetic field. In the early years of kinematic dynamo research, very little was known about the conditions of the solar interior and researchers were free to assume any interior velocity field that would give a good match with observations. Our knowledge of the interior physics has increased dramatically in the last few years due to the development of helioseismology and various kinds of numerical simulations. These developments are putting tight constraints on the types of dynamo model which can be allowed, although we are still far from evolving a 'standard' model of the solar dynamo. Lack of space unfortunately compels us to omit any discussion of important nonlinear processes and fluctuations around the mean (irregularities of the solar cycle, field concentration in flux tubes, etc.).

Let us begin by summarizing the relevant observational data. The most important observation is the equatorward migration of the belt of sunspots, which leads to the well-known butterfly diagram. We believe that the sunspots are produced by the magnetic buoyancy of a strong toroidal magnetic field underneath the Sun's surface - an idea first propounded by Parker (1955a). Hence, the butterfly diagram implies an equatorward migration of the subsurface toroidal field. Although the sunspots are the regions of strongest magnetic field on the solar surface, there 
definitely exist magnetic fields outside sunspots. In low-resolution magnetograms, one finds patches of unipolar field with magnitude of the order $1 \mathrm{G}$ propagating poleward. Even when averaged over latitude, one finds predominantly one polarity in a belt of latitudes and these latitude belts move poleward (see Wang et al. 1989 and references therein). The most plausible explanation for the weak field outside sunspots is that this is a manifestation of the poloidal magnetic field, which plays a crucial role in dynamo theory. The early dynamo theorists neglected the weak field outside sunspots and focused their attention on the butterfly diagrams of sunspots. It is now becoming clear that the weak field outside sunspots gives us valuable clues about the dynamo process and a dynamo model should attempt to explain its behaviour in addition to explaining the butterfly diagram.

\section{Fundamentals of dynamo theory}

The basic theory of the turbulent dynamo was developed in a classic paper by Parker (1955b) and then put on a firm mathematical basis by Steenbeck et al. (1966). The central idea is that the poloidal and the toroidal components of magnetic field sustain each other, drawing the energy from the reservoir of kinetic energy of motion. The differential rotation can stretch out the poloidal field lines to produce the toroidal field. On the other hand, the toroidal field is twisted by helical turbulence to give rise to the poloidal field again (a process now known as the $\alpha$-effect). Basics of turbulent dynamo theory can be found explained in Choudhuri (1998).

If we assume axisymmetry and use spherical coordinates, the magnetic field can be written as

$$
\mathbf{B}=B \mathbf{e}_{\phi}+\nabla \times\left(A \mathbf{e}_{\phi}\right)
$$

where $B(r, \theta)$ and $A(r, \theta)$ correspond to the toroidal and the poloidal components respectively. Let us write the velocity field in the following way

$$
\mathbf{v}=\mathbf{v}_{p}+r \sin \theta \Omega \mathbf{e}_{\phi},
$$

where $\Omega(r, \theta)$ is the angular velocity and $\mathbf{v}_{p}(r, \theta)$ corresponds to any circulation in the meridional plane. If the magnetic and the velocity fields are written as in (1) and (2), then the turbulent dynamo theory leads to the following equations for the evolution of poloidal and toroidal components:

$$
\begin{gathered}
\frac{\partial A}{\partial t}+\frac{1}{s}\left(\mathbf{v}_{p} \cdot \nabla\right)(s A)=\eta\left(\nabla^{2}-\frac{1}{s^{2}}\right) A+\alpha B, \\
\frac{\partial B}{\partial t}+s\left(\mathbf{v}_{p} . \nabla\right)\left(\frac{B}{s}\right)=\eta\left(\nabla^{2}-\frac{1}{s^{2}}\right) B+s\left(\mathbf{B}_{p} . \nabla\right) \Omega,
\end{gathered}
$$

where $s=r \sin \theta$ and $\eta$ is the diffusivity. The last terms in the above two equations are the dynamo source terms. The last term in (4) corresponds to the generation of toroidal field by the stretching of poloidal field $\mathbf{B}_{p}=\nabla \times\left(A \mathbf{e}_{\phi}\right)$ due to differential rotation, whereas the last term in (3) incorporates the $\alpha$-effect, i.e. the twisting of toroidal field lines by helical turbulence to produce the poloidal component. In the kinematic dynamo approach, one essentially solves (3) and (4) after specifying some 
'reasonable' distribution of $\alpha, \Omega, \eta$ and $\mathbf{v}_{p}$. Most calculations until recently did not include any meridional circulation, i.e. $\mathbf{v}_{p}$ was taken as 0 . The other parameters could not be specified completely arbitrarily. It was found (Parker 1955b) that the kinematic dynamo equations admit a wave-like solution which propagates equatorward only if the dynamo criterion

$$
\alpha \frac{\partial \Omega}{\partial r}<0
$$

is satisfied in the northern hemisphere of the Sun. Since we believe that the equatorward migration of the sunspot belt results from a dynamo wave propagating towards the equator, early dynamo theorists used to choose their parameters in such a way that the inequality (5) was satisfied.

\section{Historical development of solar dynamo models}

If one looks at the historical development of solar dynamo models, then one can clearly discern three distinct phases. In the first phase during the approximate period $\approx 1965-1980$, most dynamo models assumed that the two main ingredients of the dynamo process - the production of toroidal field by differential rotation and the production of poloidal field by $\alpha$-effect-both took place in the convection zone of the Sun. During the second phase lasting over $\approx 1980-1995$, it was assumed that both of the above-mentioned main ingredients of the dynamo process occurred in a thin layer at the base of the convection zone. Models developed in the third phase beginning around 1995 will be discussed in the next section.

Since the dynamo process is ultimately powered by the kinetic energy of motion, it was natural to assume in the beginning that the whole dynamo process takes place in the convection zone where kinetic energy is most abundant. The researchers of the first phase (Steenbeck \& Krause 1969; Roberts 1972; Köhler 1973; Yoshimura 1975; Stix 1976) were able to produce reasonable-looking butterfly diagrams by solving (3)-(4) in the convection zone of the Sun, provided the inequality (5) was satisfied. Soon it started becoming clear that magnetic buoyancy would be particularly destabilizing in the body of the convection zone and remove any magnetic flux from there rather quickly, without allowing time for amplification by the dynamo (Parker 1975). Several authors (Spiegel \& Weiss 1980; van Ballegooijen 1982; Parker 1987; van Ballegooijen \& Choudhuri 1988) suggested ways of suppressing magnetic buoyancy at the bottom of the convection zone. This led to the idea that the whole dynamo process takes place in a thin layer at the base of the convection zone. When helioseismology found a layer of concentrated shear at the base of the convection zone, this idea found further support. Several authors in the second phase of dynamo research explored the idea of the dynamo operating in a thin layer (Gilman et al. 1989; Schmitt \& Schüssler 1989; Choudhuri 1990; Belvedere et al. 1991; Rüdiger \& Brandenburg 1995).

If the dynamo operates at the base of the convection zone and the strong toroidal field is produced there, then this field has to rise through the convection zone to produce sunspots. Choudhuri \& Gilman (1987) showed that the Coriolis force plays a very important role in this problem, trying to divert the rising flux to the poleward direction. Subsequent simulations of the flux rise made it clear that the toroidal field 
at the base of the convection zone has to be about $10^{5} \mathrm{G}$ (one order larger than the equipartition value) if we are to explain various surface features of sunspots (Choudhuri 1989; D'Silva \& Choudhuri 1993; Fan et al. 1993). Since there is a shear layer at the base of the convection zone, most researchers still believe that the toroidal magnetic field is produced there. If this toroidal field is as strong as $10^{5} \mathrm{G}$, then it would completely quench the $\alpha$-effect and the traditional scenario of the dynamo process would definitely not work. It has been suggested that the buoyancy instability of this strong toroidal field itself may give rise to configurations similar to what was believed to be produced by the traditional $\alpha$-effect (Ferriz-Mas et al. 1994). Based on such ideas, some efforts have been made to develop new types of interface dynamo models (Parker 1993; Charbonneau \& MacGregor 1997). Section 4 describes a different school of thought to tackle this problem of strong toroidal field, which we call the third phase of solar dynamo research.

\section{Revival of interest in Babcock-Leighton models}

When the active regions on the solar surface decay, they spread some magnetic flux around. The leading sunspot in a bipolar pair is usually found at a lower latitude, due to the action of the Coriolis during the rise (D'Silva \& Choudhuri 1993). It was pointed out by Babcock (1961) and Leighton (1969) long time ago that the decay of such a tilted bipolar active region contributes to the poloidal field. This effect was not included in most of the detailed dynamo models during the first and second phases, when the primary emphasis was on explaining the equatorward migration of the sunspot belt. The poleward migration of the weak field was explained by assuming that this is the poloidal component which is carried by a meridional circulation flowing towards the pole near the solar surface (Wang et al. 1989; Dikpati \& Choudhuri 1994; Choudhuri \& Dikpati 1999).

Since traditional $\alpha$-effect would be inoperative on a $10^{5} \mathrm{G}$ strong toroidal field at the base of the convection zone, some theorists are now exploring the BabcockLeighton idea that the poloidal field is produced near the solar surface by the decay of active regions (Choudhuri et al. 1995; Durney 1995, 1997; Dikpati \& Charbonneau 1999; Nandi \& Choudhuri 2000). One way of incorporating this effect in dynamo calculations is to take the $\alpha$ coefficient concentrated near the surface, while the shear layer is taken at the base of the convection zone. A meridional circulation which is poleward near the surface and equatorward in the interior has to play an important role in these dynamo models. This circulation brings down the poloidal field created at the surface to the base of the convection zone where it can be stretched by the differential rotation. One difficulty with such models is that the $\alpha$ coefficient arising out of the decay of active regions has to be positive, whereas $\partial \Omega / \partial r$ at lower latitudes is also positive. It may appear at the first sight that this would violate the dynamo criterion (5) and the dynamo waves would propagate in the poleward direction. It has been shown by Choudhuri et al. (1995) that an equatorward propagation may still be possible in this situation if meridional circulation plays a very significant role-the time scale of meridional circulation being less than the time scale of diffusion across the convection zone. This result shows that this new type of model is viable and such models are now being investigated in detail by several groups. 


\section{Conclusion}

It appears that the kinematic dynamo models developed in the third phase may be free from the difficulties of the earlier models, while explaining the behaviour of both sunspots and weak magnetic fields in a unified way. We are, however, still far from developing a model which can explain all the different aspects of observation in detail.

It may be mentioned that in this review we have concentrated on those kinematic calculations which attempt to model the regularities of solar cycle. A very important problem of kinematic dynamo research is to model the irregularities of solar cycle. Although the present author has worked in this field and it is a subject close to his heart, the limitation of space made it impossible even to touch upon this subject. The reader may turn to a bigger review by the present author (Choudhuri 1999) for a discussion of some other aspects which are left out here.

\section{References}

Babcock, H.W. 1961, Astrophys. J., 133, 572.

Belvedere, G., Lanzafame, G., Proctor, M.R.E. 1991, Nature, 350, 481.

Charbonneau, P., MacGregor, K.B. 1997, Astrophys. J., 486, 502.

Choudhuri, A.R. 1989, Solar Phys., 123, 217.

Choudhuri, A.R. 1990, Astrophys. J., 355, 733.

Choudhuri, A.R. 1998, The Physics of Fluids and Plasmas: An Introduction for Astrophysicists (Cambridge University Press).

Choudhuri, A.R. 1999, Curr. Sci., 77, 1475.

Choudhuri, A.R., Dikpati, M. 1999, Solar Phys., 184, 61.

Choudhuri, A.R., Gilman, P.A. 1987, Astrophys. J., 316, 788.

Choudhuri, A.R., Schüssler, M., Dikpati, M. 1995, Astr. Astrophys., 303, L29.

Dikpati, M., Charbonneau, P. 1999, Astrophys. J., 518, 508.

Dikpati, M., Choudhuri, A.R. 1994, Astr. Astrophys., 291, 975.

D’Silva, S., Choudhuri, A.R. 1993, Astr. Astrophys., 272, 621.

Durney, B.R. 1995, Solar Phys., 160, 213.

Durney, B.R. 1997, Astrophys. J., 486, 1065.

Fan, Y., Fisher, G.H., DeLuca, E.E. 1993, Astrophys. J., 405, 390.

Ferriz-Mas, A., Schmitt, D., Schüssler, M. 1994, Astr. Astrophys., 289, 949.

Gilman, P.A., Morrow, C.A., DeLuca, E.E. 1989, Astrophys. J., 338, 528.

Köhler, H. 1973, Astr. Astrophys., 25, 467.

Leighton, R.B. 1969, Astrophys. J., 156, 1.

Nandi, D., Choudhuri, A.R. 2000, Astrophys. J., submitted.

Parker, E.N. 1955a, Astrophys. J., 121, 491.

Parker, E.N. 1955b, Astrophys. J., 122, 293.

Parker, E.N. 1975, Astrophys. J., 198, 205.

Parker, E.N. 1987, Astrophys. J., 312, 868.

Parker, E.N. 1993, Astrophys. J., 408, 707.

Roberts, P.H. 1972, Phil. Trans. Roy. Soc., 272, 663.

Rüdiger, G., Brandenburg, A. 1995, Astr. Astrophys., 296, 557.

Schmitt, D., Schüssler 1989, Astr. Astrophys., 223, 343.

Spiegel, E.A., Weiss, N.O. 1980, Nature, 287, 616.

Steenbeck, M., Krause, F. 1969, Astr. Nachr., 291, 49.

Steenbeck, M., Krause, F., Rädler, K.H. 1966, Z. Naturfor., 21a, 1285.

Stix, M. 1976, Astr. Astrophys., 47, 243.

van Ballegooijen, A.A. 1982, Astr. Astrophys., 113, 99.

van Ballegooijen, A.A., Choudhuri, A.R. 1988, Astrophys. J., 333, 965.

Wang, Y.-M., Nash, A.G.; Sheeley, N.R. 1989, Astrophys. J., 347, 529.

Yoshimura, H. 1975, Astrophys. J. Suppl., 29, 467. 